\title{
Capacidad de los servicios de salud institucionales para la atención de los usuarios de la Clínica de Estomatología de la Unidad Médico Didáctica de la Universidad Autónoma de Aguascalientes, según su zona de residencia
}

García Reyna Roberto Arturo*, Rosas Cabral Alejandro**, Prieto Macías Jorge**, Mendoza Tijerina Jaime Adrián***, Díaz-Alfaro Lizbeth*, Zertuche García Vania****

\section{Resumen}

- Las enfermedades orales en México y en el mundo son un importan-

- te problema de salud pública y afecta principalmente a los grupos

- socialmente más desfavorecidos y marginados. Objetivo. Conocer las

- necesidades asistenciales del usuario de la Clínica de Estomatología

- de la Universidad Autónoma de Aguascalientes a través de la historia

- natural de la enfermedad de los órganos dentales y su lugar de resi-

- dencia. Metodología. Se realizó un estudio descriptivo, observacional

y de corte transversal. Se registraron los dientes cariados, restaura-

dos y extraídos de la población en estudio. Resultados. Un total de

392 pacientes mayores de 20 años fueron revisados, de los cuales

223 fueron mujeres (56.9\%) y 169 (43.1\%) hombres. Los hombres

mostraron una media de $8.48 \pm 2.40$ dientes cariados; $2.41 \pm 1.82$

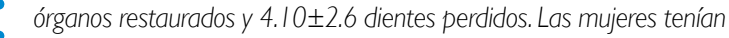

- un promedio de 8.62 \pm 1.88 dientes cariados; $4.06 \pm 1.73$ dientes res-

- taurados y 4.52 3.64 dientes faltantes. Existen sólo 18 dentistas en

- las unidades de los diferentes sistemas de salud gubernamental, para

- brindar atención odontológica a 446,546 habitantes de la zona orien-

- te de la Ciudad de Aguascalientes, con una proporción resultante

- de 24,808.1I habitantes por cada dentista. Conclusiones. Encontra-

- mos una gran discrepancia entre los dientes cariados y restaurados;

- el edentulismo es mayor al reportado por SSA y se tiene un bajo

- acceso al servicio de salud dental; la cobertura dental del sector oficial

- en la zona oriente de la ciudad de Aguascalientes es insuficiente y

- existe una baja cultura en la población de autocuidado que impacta

- su salud y economía. LUXMÉDICA, AÑO 14, NÚMERO 40, ENERO-ABRIL

- 2019, PP 29-39.

Palabras Clave: Políticas de salud, salud pública, accesibilidad, salud y economía

\section{Abstract}

Oral diseases in Mexico and the world are an important public health problem and mainly affect the most socially disadvantaged and marginalized groups. Objective: To know the healthcare needs of the user of the Stomatology Clinic of the Universidad Autonoma de Aguascalientes, through the natural history of the disease of the dental organs and their place of residence. Methods: A descriptive, observational and cross-sectional study was carried out. The decayed, restored and extracted teeth of the study population were recorded. Results: A total of 392 patients older than 20 years were evaluated, of which 223 were women $(56.9 \%)$ and $169(43.1 \%)$ men. The men showed an average of $8.48 \pm 2.40$ decayed teeth; $2.41 \pm 1.82$ organs restored, and 4.10 \pm 2.6 teeth lost. The women had an average of $8.62 \pm 1.88$ decayed teeth; $4.06 \pm 1.73$ restored teeth, and $4.52 \pm 3.64$ missing teeth. There are only 18 dentists in the units of the different governmental health systems, to provide dental care to 446,546 inhabitants of the eastern area of the city of Aguascalientes, with a resulting proportion of 24,808.1 I inhabitants per dentist. Conclusions: We found a large discrepancy between decayed and restored teeth; edentulism is greater than that reported by the state health ministry, and there is low access to dental health service; dental coverage of the official sector in the eastern area of the city of Aguascalientes is insufficient and there is a low culture of self-care in the population which impacts their health and economy. LUXMÉDICA, AÑO 14, NÚMERO 40, ENERO-ABRIL 2019, PP 29-39.

Keywords: health policies, public health, accessibility, health and economy

Departamento de Estomatología, Centro de Ciencias de la Salud, Universidad Autónoma de Aguascalientes

** Departamento de Medicina, Centro de Ciencias de la Salud, Universidad Autónoma de Aguascalientes.

*** Área de Odontología Preventiva y Social de la Facultad de Odontología de la Universidad Autónoma de Nuevo León

**** Estudiante de la carrera de Medicina del Centro de Ciencias de la Salud de la Universidad Autónoma de Aguascalientes

Fecha de recibido: 25 de octubre 2018

Fecha de aceptación: 26 de noviembre 2018

Autor para correspondencia: Dr. Alejandro Rosas Cabral. Departamento de Medicina, Edifico 107, planta alta. Centro de Ciencias de la Salud, Universidad Autónoma de Aguascalientes, Ave. Universidad \#940, Ciudad Universitaria, CP 20131, Aguascalientes, Aguascalientes, México. Teléfono 9107400, extensión 53014. Correo electrónico drrosascabral@gmail.com 


\section{Introducción}

De acuerdo a la Organización Mundial de la Salud (OMS) y con el Perfil Epidemiológico de la Salud Bucal en México 2015, las enfermedades bucales son consideradas como uno de los problemas de salud pública más importantes, debido a su elevada prevalencia e incidencia en diversas regiones del mundo y en este país, por lo que representan una importante carga para la población, afectando sobre todo a los más desfavorecidos y marginados socialmente. ${ }^{1}$

Se reconoce que los principales problemas de salud bucal en nuestra población son la caries dental y la enfermedad periodontal; ambas tienen un alto impacto en la calidad de vida de quien las padece, como lo menciona Spencer en su estudio acerca del Perfil de Impacto de Salud Oral (OHIP), basado en una recopilación de 49 artículos sobre la percepción de la salud oral y su relación con la calidad de vida, tomando en cuenta la limitación funcional, el dolor físico y el malestar psicológico, cuantificando las consecuencias sociales de la enfermedad a través de las limitaciones en la vida diaria y la calidad de vida. Tal como se observa en pacientes que, por pérdida dental, ven limitada la función masticatoria, la fonética y la confianza por pérdida estética, importante para la socialización. ${ }^{2}$

Además del impacto antes mencionado se debe tomar en cuenta que existe una robusta evidencia que ha asociado enfermedades sistémicas tales como: endocarditis, ${ }^{3}$ enfermedad coronaria, ${ }^{4,5}$ infarto, ${ }^{6}$ hipertensión, ${ }^{7}$ diabetes mellitus ${ }^{8,9}$, bajo peso al nacer, nacimientos prematuros, ${ }^{10,11}$ cáncer $^{12}$ y diabetes gestacional ${ }^{13}$ a la flora patogénica de la cavidad bucal resaltando la importancia de su adecuada y pronta atención.

De acuerdo con lo anterior, para favorecer el estado de salud de la población, la OMS establece que: "la Atención Primaria de salud es la asistencia sanitaria esencial accesible a todos los individuos y familias de la comunidad a través de medios aceptables para ellos, con su plena participación y a un costo asequible para la comunidad y el país". ${ }^{14}$ Es el núcleo del sistema de salud de un país y forma parte integral del desarrollo socioeconómico general de esa comunidad. ${ }^{15}$

De esto se destacan dos aspectos importantes:

a) Que la población debe ser susceptible de recibir, como parte de esa atención primaria, un enfoque adecuado en la salud bucodental mediante el desarrollo de programas destinados a preservar y favorecer el desarrollo integral y económico de la población

b) Que para que estos programas puedan ser aplicados, se debe contar con recursos suficientes y que, además, su costo debe ser accesible para la población.

Sin embargo, para el diseño de sistemas de atención a la salud dental, uno de los elementos principales a tomar en cuenta es determinar las necesidades de atención de la enfermedad, es decir, su morbilidad; una vez que se dispone de datos confiables acerca de la prevalencia, la incidencia y su historia natural, es posible es- 
tablecer una estrategia de atención con la cual hacer frente al problema y sustentar un sistema de salud dental adecuado. ${ }^{16}$

Dentro de las estrategias a seguir, se debe considerar además el acceso y el uso efectivo de los servicios de salud $y$, para ello, éstos se deben dimensionar en cuanto a su disponibilidad, calidad y accesibilidad.

Entendiéndose la dimensión de la disponibilidad a la suficiencia de infraestructura; como calidad a que la infraestructura de los establecimientos, así como que los bienes y servicios de salud sean apropiados a las necesidades de atención de los pacientes, y como accesibilidad a que no existan barreras u obstáculos para el obtención de los servicios de salud, sean éstos de tipo físico, económico o de información sobre la ubicación del servicio. Una de las barreras más evidentes de la accesibilidad son las barreras organizacionales que influyen sobre los diferimientos y tiempos de espera para el paciente, debido a una utilización ineficiente de los recursos o a lineamientos inadecuados para otorgar la atención. ${ }^{17-20}$

La adecuada aplicación de los recursos físicos y humanos debe ser orientada por estudios tendientes a conocer las necesidades de atención de la población, saber dónde habita, los recursos de salud tanto físicos como humanos con que se cuenta, si son suficientes y accesibles, así como las implicaciones económicas y sociales de la enfermedad, como lo refiere Maupome. ${ }^{16}$

En México y en Aguascalientes, la información acerca del impacto económico, social y en la salud integral que ocasionan los principales problemas bucales que presenta la población, aunado a que la atención es limitada, afecta las acciones a seguir para el establecimiento de programas que los resuelvan adecuadamente. ${ }^{21-23}$

El presente estudio tiene por objetivo conocer las necesidades de atención, basados en la historia natural de la enfermedad acumulativa de los órganos dentarios, desde la presencia de caries hasta su pérdida, de los usuarios del servicio de Diagnóstico Estomatológico de la Unidad Medico Didáctica (UMD) de la Universidad Autónoma de Aguascalientes (UAA), considerando además su zona de residencia y la capacidad de los servicios de salud oficiales, que les prestan atención.

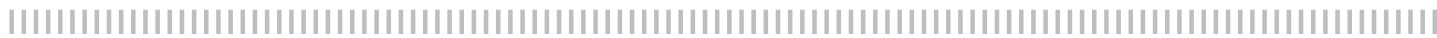

\section{Metodología}

El presente es un trabajo observacional, descriptivo y transversal en el que se estudió a los pacientes que acudieron al servicio de Diagnóstico de la Unidad Médico Didáctica de la UAA los días martes y jueves por la mañana y miércoles y viernes por la tarde durante el periodo de agosto a diciembre del 2015. Para la evaluación de la caries dental, se utilizaron los criterios de la Organización Mundial de la Salud (OMS), mediante el empleo de un explorador del número 2.

Se cuantificaron las restauraciones inmediatas (resina y amalgama) y las mediatas (coronas e incrustaciones) para el tratamiento de las cavidades cariosas presentes al momento de la exploración. Los dientes no presentes al momento del examen se tomaron como perdidos sin importar si se perdieron por enfermedad periodontal o caries o por presentar agenesia. Se utiliza para este estudio la evaluación de las piezas dentales en forma de promedio debido a que ésta es la norma internacional para su reporte.Para determinar la capacidad de los servicios de salud dentales institucionales, registramos el número de odontólogos y pasantes de odontología que laboran en las diferentes unidades del sector salud (IMSS, ISSSTE, SSA), en los diferentes turnos y se determinó también el número de pacientes que atienden por turno. Para determinar la población total cubierta en esa zona de la ciudad se obtuvieron los datos proporcionados por el INEGI para el Censo de 2010. Para el análisis estadístico, se emplearon medidas de tendencia central, índices y proporciones y prueba de $\mathrm{T}$ de Student, para comparación de medias. 


\section{Resultados}

Se revisaron un total de 392 pacientes, de los cuales 223 fueron mujeres (56.9\%) y $169(43.1 \%)$ hombres. Cabe mencionar que los pacientes examinados correspon- den al $25.06 \%$ de la población que solicitó servicio de diagnóstico en la Clínica de Estomatología del mes de agosto a noviembre de 2015 (Tabla 1).

\section{Tabla I}

Distribución por edad y sexo de pacientes atendidos en el servicio de Diagnóstico Estomatológico de la UMD de la UAA en el segundo semestre de 2015.

\begin{tabular}{|c|c|c|c|c|c|}
\hline Edad (años) & Hombres & & Mujeres & & Total \\
\hline & $\mathrm{n}$ & $\%$ & $\mathrm{n}$ & $\%$ & \\
\hline 13 a 20 & 36 & 21.30 & 40 & 17.93 & 76 \\
\hline 21 a 30 & 37 & 21.89 & 51 & 22.83 & 88 \\
\hline 31 a 40 & 24 & 14.20 & 40 & 17.93 & 64 \\
\hline 41 a 50 & 22 & 13.01 & 39 & 17.48 & 61 \\
\hline 51 a 60 & 22 & 13.01 & 28 & 12.55 & 50 \\
\hline 61 a 75 & 28 & 16.56 & 25 & 11.21 & 53 \\
\hline TOTAL & $\mathbf{1 6 9}$ & $\mathbf{4 3 . 1 1}$ & $\mathbf{2 2 3}$ & $\mathbf{5 6 . 8 8}$ & $\mathbf{3 9 2}$ \\
\hline
\end{tabular}

En el grupo de hombres el promedio de dientes presentes fue en total 4,338 órganos dentarios, con una media de $24.70 \pm$ 3.97 por paciente; 1,408 órganos dentarios cariados $(32.45 \%)$, con una media de $8.48 \pm 2.40$ dientes cariados por paciente; un total de 397 órganos obturados (9.15
\%) con una media $2.41 \pm 1.82$ por paciente y 694 dientes perdidos con una media de $4.10 \pm 2.6$ por paciente. Los promedios de dientes presentes, cariados, perdidos y obturados por grupos de edad (Tabla 2)

\section{Tabla 2}

Distribución de dientes con caries, restaurados y perdidos, según grupo de edad en 169 hombres que acudieron al servicio de diagnóstico clínico estomatológico de la Unidad Médico Didáctica de la UAA en el segundo semestre de 2015.

\begin{tabular}{|c|c|c|c|}
\hline Edad (años) & Dientes cariados & Dientes perdidos & Dientes restaurados \\
\hline 13 a 20 & $6.05 \pm 3.97$ & $0.44 \pm 0.99$ & $0.77 \pm 1.41$ \\
\hline 21 a 30 & $9.59 \pm 4.95$ & $1.16 \pm 1.57$ & $1.29 \pm 2.13$ \\
\hline 31 a 40 & $11.67 \pm 5.0$ & $1.58 \pm 2.28$ & $2.00 \pm 3.25$ \\
\hline 41 a 50 & $10.77 \pm 6.86$ & $4.22 \pm 3.53$ & $5.18 \pm 4.32$ \\
\hline 51 a 60 & $5.45 \pm 4.16$ & $7.05 \pm 5.69$ & $3.59 \pm 4.28$ \\
\hline 61 a 75 & $6.84 \pm 5.85$ & $11.68 \pm 10.58$ & $1.72 \pm 3.32$ \\
\hline > 76 & $5.0 \pm 4.01$ & $19.0 \pm 11.27$ & $1.00 \pm 1.73$ \\
\hline
\end{tabular}


Como también se puede observar en la Tabla 2, los grupos de edad de 21 a 30 , de 31 a 40 y de 41 a 50 años, representan al $49.1 \%$ de la muestra, y tienen una media entre estos tres grupos de 10.67 lesiones cariosas por persona, por lo que requieren de una cantidad mayor de obturaciones, de las cuales los pacientes solo se han atendido un promedio de 2.83 dientes cariados.

En el grupo de mujeres, el promedio de dientes presentes fue de 5,961 con una media de $25.73 \pm 2.97$ dientes por persona por paciente; 1,943 dientes cariados con una media de $8.62 \pm 1.88$ por paciente; 857 dientes obturados, con una media $4.06 \pm 1.73$ dientes por paciente y un total de 856 dientes perdidos con una media de $4.52 \pm 3.64$ por paciente. Los promedios de dientes presentes, cariados, perdidos y obturados por grupos de edad en mujeres (Tabla 3).

\section{Tabla 3}

Distribución de dientes con caries, restaurados y perdidos según grupo de edad en mujeres que acudieron al servicio de diagnóstico estomatológico de la UMD de la UAA en el segundo semestre de 2015.

\begin{tabular}{|c|c|c|c|}
\hline Edad (años) & Dientes cariados & Dientes perdidos & Dientes restaurados \\
\hline 13 a 20 & $7.07 \pm 4.46$ & $0.90 \pm 1.42$ & $1.50 \pm 2.83$ \\
\hline 21 a 30 & $7.76 \pm 4.27$ & $1.33 \pm 1.64$ & $2.17 \pm 2.65$ \\
\hline 31 a 40 & $11.15 \pm 5.06$ & $2.85 \pm 2.41$ & $4.37 \pm 4.63$ \\
\hline 41 a 50 & $9.46 \pm 3.95$ & $4.69 \pm 3.06$ & $4.79 \pm 4.92$ \\
\hline 51 a 60 & $6.26 \pm 3.26$ & $7.75 \pm 5.37$ & $4.71 \pm 3.27$ \\
\hline 61 a 75 & $6.2 \pm 4.21$ & $10.80 \pm 8.09$ & $3.40 \pm 3.31$ \\
\hline > 76 & 0.00 & 0.00 & 0.00 \\
\hline
\end{tabular}

Los grupos de edad de 21 a 30, de 31 a 40 y de 41 a 50 años de las mujeres representan al $58.24 \%$ de la muestra, con una media entre estos tres grupos de 9.45 lesiones cariosas por persona, de las que sólo se han atendido un promedio de 3.7 dientes cariados, con una media de 5.75 lesiones cariosas por atender. No se presentó ninguna paciente mayor a 75 años.

No se encontraron diferencias estadísticamente significativas entre los grupos de hombres y mujeres excepto para el grupo de 31 a 40 años en el apartado de dientes perdidos y obturados $(p=0.026)$. Así mismo, los grupos de edad de 61 a 75 y mayores de 75 años presentan la población con mayor pérdida de órganos dentarios con una media de 15.34.

Se encontró que, de acuerdo con la zona de la ciudad de donde provienen los usuarios, el $27 \%$ correspondieron a la zona nororiente, el $27 \%$ provino de la zona suroriente, el $8 \%$ provino de la zona norponiente, el $7 \%$ a la zona sur-poniente y el $3 \%$ de la zona centro. El $25 \%$ de los pacientes provinieron de los municipios del estado; sin embargo, el $21 \%$ de éstos provinieron del municipio de Jesús María y sólo el $4 \%$ del resto de los municipios. El $3 \%$ restante provino de municipios de los estados de Jalisco y Zacatecas (Figura 1). 


\section{Procedencia de pacientes}

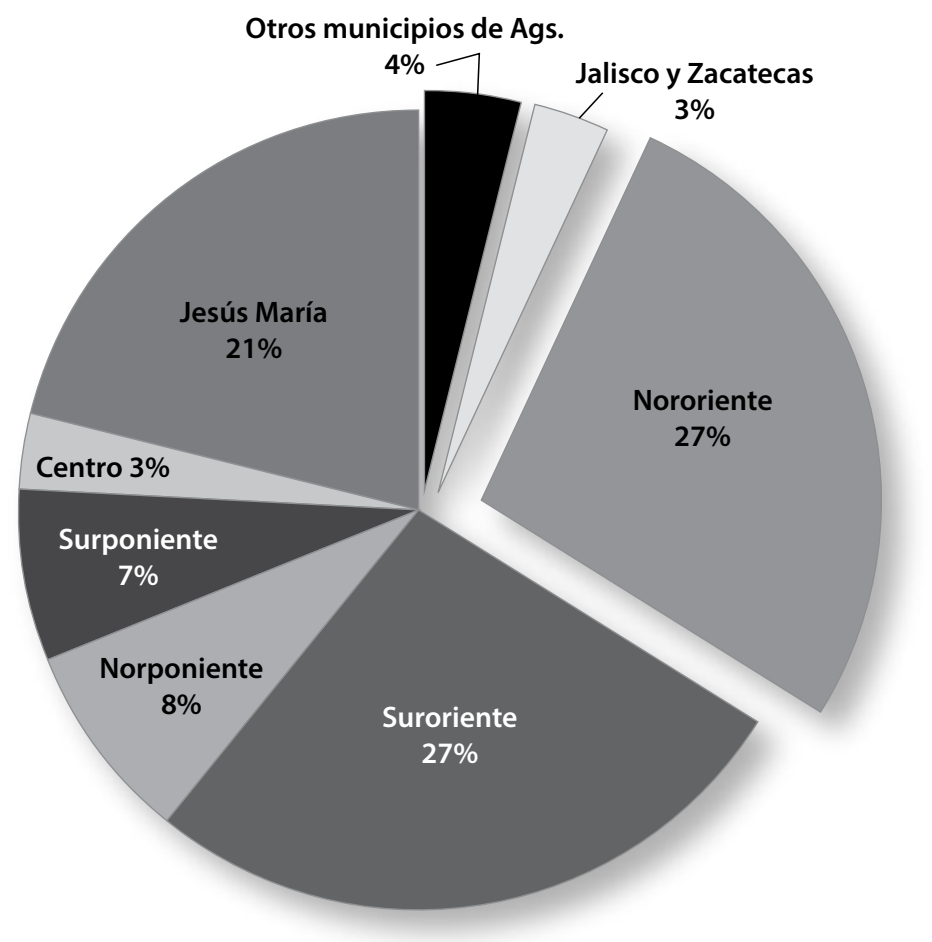

Figura 1. Distribución de los pacientes que acudieron al servicio de Diagnóstico de la Clínica de Estomatología de la Unidad Medico-Didáctica de la UAA según el sitio de su vivienda.

Según datos proporcionados por el INEGl, el 54\% de la población que acude a consulta a la clínica de Estomatología proviene de las Delegaciones Lic. Jesús Terán Peredo (zona nororiente), que está comprendida entre la salida a la ciudad de San Luis Potosí y la salida a la ciudad de Zacatecas; y la delegación José Ma. Morelos y Pavón (zona sur oriente), comprendida entre la salida a San Luis Potosí y la salida a la Ciudad de México a partir de la avenida de la Convención de 1914. Estas zonas del municipio de Aguascalientes, en el 2013, presentaron una densidad de población de 6,059.14 personas por kilómetro cuadrado (Km2) correspondiendo 4,017.576 habitantes/Km2 a la delegación Jesús Terán y 2,041.5709 habitantes $/ \mathrm{Km}^{2}$ a la delegación José Ma. Morelos.
La suma total de la población en estas delegaciones es de 446,546 habitantes, de los cuales 217,745 son hombres y 227,776 son mujeres de ellos 295,953 son mayores de 15 años y de los cuales 141,698 son hombres y 154,219 son mujeres. La zona oriente está reportada como una zona de bajos recursos económicos y de acuerdo con los datos proporcionados por el INE$\mathrm{Gl}$ en el año 2012, las delegaciones que comprende esta zona presentaron una importante proporción de beneficiarios de apoyos de los diversos programas sociales gubernamentales. Así mismo, el INEGI reporta en el censo del 2000 (es la única información con que se cuenta a estos niveles de desagregación geográfica) que en ese año el ingreso promedio para estas delegaciones se estableció en $\$ 3.76$ pesos por hora (Figura 2). 


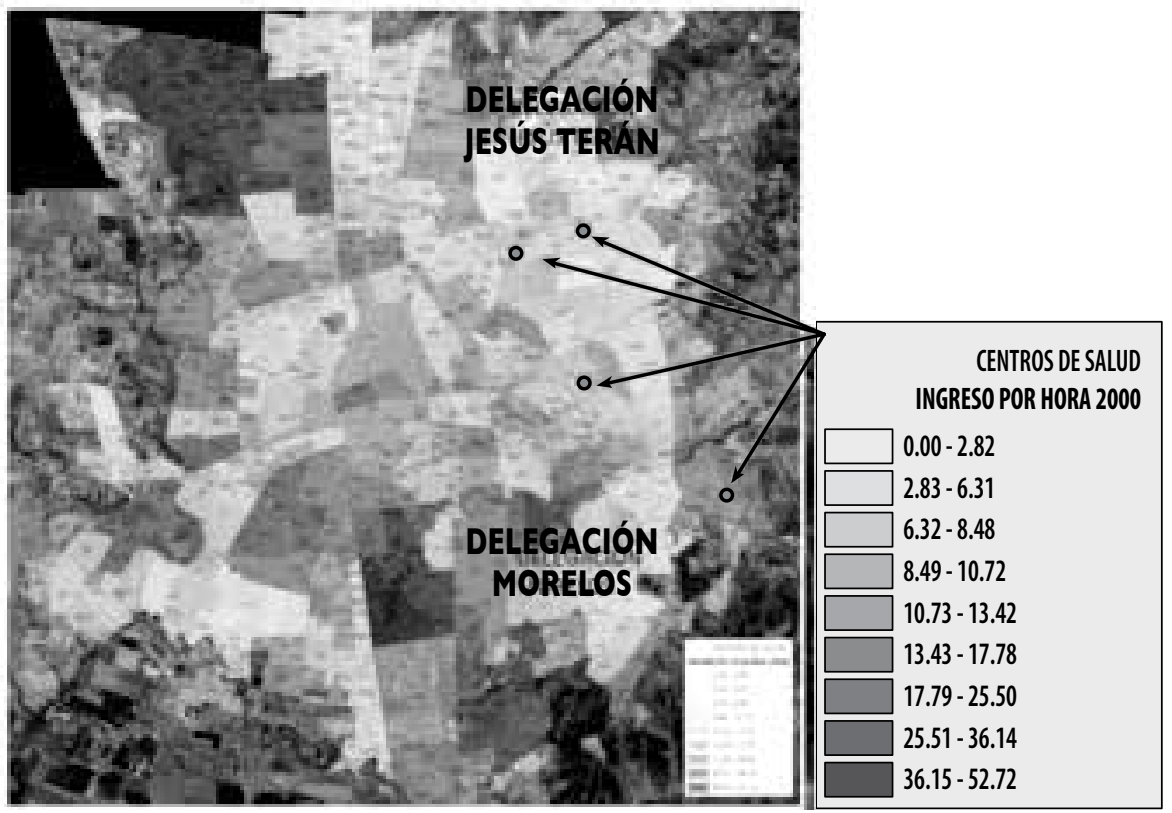

Figura 2. Mapa de ingreso por hora en pesos mexicanos y ubicación de centros de salud en la zona (fuente: INEGI, Coordinación estatal)

Para dar atención a la salud de la población en la zona nororiente y específicamente para la salud bucodental, el Instituto de Servicios de Salud de Aguascalientes (ISSEA) cuenta con los siguientes Centros de Salud Urbanos: Progreso, atendido por un Odontólogo con horario de 07:30 a 15:30 horas; Cumbres también con un Odontólogo con horario de 7:30 a 15:30 horas; Ojocaliente, con dos Odontólogos por la mañana con horario de atención de 8:00 a 14:00 horas y por la tarde con un pasante con horario de las 14:00 a las 20:00 horas y el de Guadalupe Peralta con un Odontólogo con horario de 07:30 a 15:30 y un pasante con horario de 14:00 a 20:00 horas. Cabe mencionar la reciente apertura de otra unidad de salud ubicada en Haciendas de Aguascalientes con dos Odontólogos con horarios de 8 a 14 horas $y$ de 14 a 20 horas.

El Instituto Mexicano del Seguro Social (IMSS) cuenta con la unidad de Medicina Familiar No. 9 con dos estomatólogos con horario de 07:30 a 14:00 horas y otro de 13:30 a 20:00 (Plantilla de Personal Delegacional del IMSS).

Así mismo, el ISSTE en la Clínica de Medicina Familiar cuenta en esta zona con dos Odontólogos con horarios de 8:00 a 14:00 horas y de 14:00 a 20:00 horas, con un mínimo de atención de ocho pacientes por turno.

En la zona sur oriente el ISSEA cuenta con el Centro de Salud Morelos que cuenta con un dentista de 8:00 a 15:30 y un pasante de 14:00 a 20:00 horas además de un dentista de jornada acumulada, sábados y domingos, de 08:00 a 20:00 horas.

Mientras el IMSS cuenta con la Unidad de Medicina Familiar 11 que cuenta con dos estomatólogos con horario de 07:30 a 14:00 horas y otro de 13:30 a 20:00

En el caso de los Centros de Salud Urbanos el servicio es de lunes a viernes y se reparten 9 fichas por odontólogo por turno. La atención que se ofrece es de: extracciones, recubrimientos pulpares, odontoxesis, 
profilaxis, toma de radiografías periapicales, consultas, restauraciones temporales y de resina y/o amalgama, estas últimas con un costo que va de $\$ 32$ a 86 pesos para los usuarios que no cuentan con seguro popular y sin costo para los que sí cuentan con él. En las Unidades de Medicina Familiar del IMSS se citan 18 pacientes por turno y se realizan los mismos tratamientos, excepto restauraciones de resina, sin costo para sus derechohabientes. En la Clínica de Medicina Familiar del ISSSTE se realizan los mismos tratamientos que en los Centros de Salud Urbanos. En total sólo existen 18 dentistas en las unidades de los diferentes sistemas de salud gubernamental, para brindar atención odontológica a 446,546 habitantes de la zona oriente de la Ciudad de Aguascalientes, con una proporción resultante de $24,808.11$ habitantes por cada dentista.

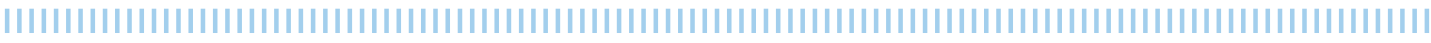

\section{Discusión}

En 1981, la OMS declaraba que el impresionante aumento en la prevalencia de la caries dental, aunado a una elevada prevalencia de las parodontopatías, provocaron que una proporción creciente de la población sufriera de ataques repetidos de intensos dolores desde la primera infancia hasta el tercero o cuarto decenio de la vida. El dolor provocaba la inmediata demanda de servicios dentales, que tendían ante todo, a aliviar esos dolores pero que contribuían en gran medida a la pérdida prematura de los dientes. ${ }^{24}$

En este estudio se encontró que el panorama no ha variado mucho, ya que hallamos que existe una amplia discrepancia entre el promedio de 2.83 dientes obturados y 10.87 dientes cariados en el grupo de hombres, y en el grupo de las mujeres 3.7 obturados y 9.47 cariados en los mismos grupos de edad, lo que indican que el estado de salud que presenta la población estudiada requiere de tratamientos de restauración con una media de 8.04 órganos dentarios por hombre y 5.77 órganos dentarios por mujer, tratamientos necesarios para evitar que se genere un mayor grado de lesión y que derive en costos más elevados para el paciente para subsanar la pérdida de órganos dentarios con tratamientos sustitutivos.

Resultados similares fueron encontrados por Caballero García en Paraguay al reportar una media de $7.0 \pm 4.5$ órganos

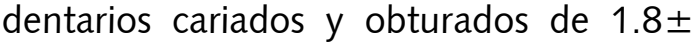

2.9. ${ }^{25}$ Los cuales también son congruentes con lo reportado por Lukes, quien en un estudio realizado en trabajadores agrícolas migrantes en el estado de Illinois, EUA con edades de los 10 a 44 años, reporta que aproximadamente el $40 \%$ tenía 6 o más superficies dentales con caries sin tratamiento y que en el grupo de 45 a 64 años este porcentaje se incrementó al $69 \%$. Reporta además, que el $51 \%$ de los inmigrantes no había buscado atención médica en el centro de salud de los trabajadores agrícolas ${ }^{26,27}$ Diferente de lo reportado por Hugoson en Suiza quien encontró una media de dientes obturados de 9.28

La elevada discrepancia encontrada por nosotros entre la mínima cantidad de procedimientos restaurativos realizados y la elevada prevalencia de caries, puede atribuirse a que la atención dental que reciben es ocasional y es congruente con lo reportado por el Departamento de Servicios Humanos y de Salud de Estados Unidos donde los integrantes del estudio de migrantes, al ser cuestionados sobre su demora en la atención, manifestaron que la principal razón fue la ausencia de dolor o incomodidad. Consecuentemente, las afecciones que podrían resolverse mediante la utilización de niveles de intervención primarios o secundarios deben, en cambio, tratarse con el objetivo de limitar la discapacidad, ${ }^{29,30}$ con el impacto económico resultante para el paciente.

Estos resultados sugieren que, al igual que lo reportado por otros autores, en esta 
población no hemos logrado implementar una cultura de auto-cuidado y prevención, tendiente a que el paciente acuda a consulta dental aun sin dolor, debido a que en la mayoría de los casos los pacientes acuden a consulta dental solo por urgencia, es decir, sólo cuando presenta molestia y el tratamiento que reciben, la mayoría de las veces, será mutilante.

Lo anterior, aunado a que no se cuenta con un programa definido de atención que permita una eficaz política pública para la atención de la salud bucodental, diseñada con base en las necesidades reales de la población. ${ }^{31}$

Asimismo, se encontró que la media de órganos dentarios perdidos que presentan el grupo de edad de 61 a 75 años de mujeres y hombres de la población, es más elevado que el que reporta, el Sistema de Vigilancia Epidemiológica de Patologías Bucales SIVEPAB 2015 a nivel nacional (promedio de dientes perdidos en mujeres de 9.25 y 7.75 en hombres). ${ }^{1}$

Sin embargo, la Secretaría de Salud reconoce que "estas cifras son inferiores a las encontradas por otros investigadores en el país, esto quizá se deba, por una parte, al tipo de población en los estudios (la fase permanente del sistema sólo examina a población usuaria de los servicios de salud) y, por otra parte, a que la utilización regular de los servicios odontológicos se asocia con el número de dientes remanentes en boca."

No obstante, los resultados son congruentes a lo reportado en un estudio realizado en Paraguay donde se reportan para el mismo grupo de edad 13.3 órganos perdidos, sin especificar género; en Suiza se reporta menos de 10 dientes perdidos en el grupo de 70 años sin especificar género; la relevancia de este dato, además de permitir conservar una adecuada función masticatoria, se basa en reportes recientes que relacionan el edentulismo como factor de riesgo para la enfermedad de Alzheimer. ${ }^{32}$

Los datos anteriores nos permiten concluir que existe un limitado acceso a la uti- lización de los servicios odontológicos y a los tratamientos restauradores en nuestra población, situación que ocasiona un mínimo contacto con el odontólogo, no teniendo oportunidad de detectar y disminuir los hábitos nocivos para la salud bucodental que ayuden a prevenir las caries, los problemas periodontales y las pérdidas dentarias prematuras.

En este estudio, el $54 \%$ de los pacientes provinieron de las Delegaciones Lic. Jesús Terán Peredo (Zona nororiente), y la delegación José Ma. Morelos y Pavón (Zona sur oriente) de la ciudad, contando el sector salud con sólo 18 odontólogos.

La OMS reporta en sus Estadísticas Sanitarias Mundiales 2013, una relación dentistas/por cada 10,000 habitantes en los siguientes países: Líbano 13.3, Brasil 11.7, Colombia 9.2, Dinamarca 8.1, Cuba 16.3, Bélgica 7.9, Canadá 5.9, Francia 6., Finlandia 7.6, Costa de Marfil 0.1 e India 0.8; considerando un promedio a nivel mundial de 2.6 dentistas por 10,000 habitantes. México no reporta para ese año su relación dentista/ 10,000 habitantes

De acuerdo a los parámetros establecidos por la OMS en la zona oriente de la Ciudad de Aguascalientes se cuenta con 2.8 odontólogos por cada 10,000 habitantes cercano al promedio mundial y por encima de países como la India o Costa de Marfil, pero muy por debajo de países con sistemas de salud más eficientes como es el caso de Cuba y los países del Norte de Europa.

En este mismo documento la OMS aclara que: "las estimaciones de la densidad de personal sanitario se refieren a la fuerza laboral sanitaria en activo, es decir, a la que está participando actualmente en el mercado de trabajo sanitario. Los datos proceden de numerosas fuentes, a saber, censos de población nacionales, encuestas de población activa y de empleo, encuestas en establecimientos sanitarios, y sistemas de información administrativa ordinaria. Dada esta gran diversidad de fuentes, los datos varían mucho en cobertura y calidad 
y es probable que las cifras estimadas sean superiores o inferiores a las reales."

Asimismo la OMS considera que el número de odontólogos por habitante ideal, varía de acuerdo con las necesidades de atención a la población y con los recursos disponibles, esto hace necesario conocer qué problema de salud presenta la población, dónde habita y reconocer los factores que inciden en su estado de salud bucodental para establecer estrategias para su adecuada atención.

Con el número de odontólogos por cada 10,000 habitantes que presenta la zona oriente de la ciudad de Aguascalientes es necesario optimizar los recurso del sector oficial con procedimientos de admisión y atención eficientes que facilite la accesibilidad de los pacientes sin lapsos de espera de atención prolongados, disminuir los tratamientos mutilantes y establecer políticas a largo plazo tendientes a que la población comprenda que la salud de la cavidad oral, repercute no sólo en el ámbito de la adecuada función masticatoria, socialización (autoestima) y bienestar físico (dolor) sino también su repercusión en la salud sistémica.

\section{Conclusiones}

Con los recursos tanto físicos como humanos con que actualmente cuentan las instancias públicas de salud, es improbable atender a una población numerosa para disminuir la discrepancia entre el número de dientes cariados y obturados, así como ofrecer tratamiento de segundo nivel tendiente a evitar en lo posible los tratamientos mutilantes. Se deben optimizar los lineamientos institucionales para que los trámites de atención a los pacientes sean ágiles y accesibles y redunden en una atención oportuna, eficiente y eficaz de los problemas de salud bucal que presenta la población.

\section{Bibliografía}

1. Resultados del Sistema de Vigilancia Epidemiológica de Patologías Bucales SIVEPAB 2015.

2. Slade G, Spencer J. Development and evaluation of the oral health impact profile. Community Dent Health. 1994; 11(1):3-11.

3. Cotti E. Apical periodontitis and cardiovascular diseases: previous findings and ongoing research. International Endodontic Journal. 2015; 48(10):92632.

4. Palm F. Biomarkers of periodontitis and inflammation in ischemic stroke: A case-control study. Innate Immunology. 2014; 20(5):511-8.

5. Hosomi N. Association of serum anti-periodontal pathogen antibody with ischemic stroke. Cerebrovascular Disease 2012; 34(5-6):385-92.

6. Khalid W. Role of endothelin-1 in periodontal diseases: A structured review. Indian J Dental Research. 2016 May-Jun; 27(3):323-33.

7. Rebelo MA. Low Social Position, Periodontal Disease and Poor Oral Health-Related Quality of Life in Adults with Systemic Arterial Hypertension. J Periodontol. 2016; 29:1-16.
8. Hong JW. The Prevalence and Associated Factors of Periodontitis According to Fasting Plasma Glucose in the Korean Adults: The 2012-2013 Korea National Health and Nutrition Examination Survey. Medicine (Baltimore). 2016 Apr; 95(14):e3226.

9.- Sasaki H. Interrelationship Between Periapical Lesion and Systemic Metabolic Disorders. Current Pharmaceutical Desing.2016; 22(15):2204-15.

10.-Tellapragada C. Risk Factors for Preterm Birth and Low Birth Weight Among Pregnant Indian Women: A Hospital-based Prospective Study. J Preventive Medicine and Public Health.2016; 49(3):165-75.

11.-Mahendra J. Evidence Linking the Role of Placental Expressions of Peroxisome Proliferator-Activated Receptor- $\square$ and Nuclear Factor-Kappa B in the Pathogenesis of Preeclampsia Associated With Periodontitis. Journal of Periodontol. 2016; 87(8):962-70.

12.-Zeng XT. Xia LY2, Zhang YG3, Li S1, Leng WD2, Kwong JS1.Periodontal Disease and Incident Lung Cancer Risk: A Meta-Analysis of Cohort Studies. J Periodontology. 2016; 13:1-13.

13.-García-Reyna RA, Terrones Saldivar MC, MalacaraRosas AM, Zaragoza-Velásquez N, Rosas-Cabral A y Gutiérrez Campos R. Prevalencia de los genotipos 
fimA II y fimA IV de Porphyromonas gingivalis en un grupo de mujeres mexicanas con diabetes gestacional en la región centro de México. Revista Clínica de Periodoncia, Implantologia y Rehabilitacion Oral. 2014; 7(2):85-92

14.-Organización Mundial de la Salud. La financiación de los sistemas de salud: el camino hacia la cobertura universal. Informe mundial de la salud 2010.

15.-Organización Panamericana de la Salud. Estrategia para la cobertura universal de salud. 154ava. Sesión del Comité Ejecutivo, Washington D.C., junio 2014.

16.-Maupome-Cervantes G. Prevalencia de Caries en zonas rurales y peri-urbanas marginadas. Salud Pública México. 1993; 35(4):357-367.

17.-Gulliford $M$, Figueroa-Muñoz J, Morgan M, Hughes $D$, Gibson B, Beech R, et al. What does "access to health care" mean? J Health Serv Res Policy. 2002; 7(3):186-188.

18. Consejo Nacional de Evaluación de la Política de Desarrollo Social, Indicadores de acceso y uso efectivo de los servicios de salud de afiliados al Seguro Popular, Primera edición, noviembre, 2014

19.-Fajardo-Dolci G. Acceso efectivo a los servicios de salud: operacionalizando la cobertura universal en salud. Salud Pública Méx. 2015; 57(2):180-186

20.-Millman M. Access to Health Care in America. Washington, DC: The National Academies Press, 1993.

21 Abramson JH. Community-oriented primary care. Strategy, approaches and practice: A review. Public Health Rev 1988; 16:35-98.

22 Moreno-Altamirano A. Cartas al editor. Propone aplicar un índice comunitario de fluorosis. Salud Publica Mex 2001; 43:179-81.

23.-Reisine ST. Dental health and public Policy: The social impact of dental disease. Am J Public Health 1985; 75:27-30.

24. OMS, Publicación en offset, $N^{\circ} 53$ PLANIFICACION DE SERVICIOS DE SALUD BUCODENTAL Organización Mundial de la Salud, Ginebra. 1981

25. Caballero-García CR. Salud bucodental y utilización de servicios Odontológicos. Mem. Inst. Investig. Cienc. Salud vol.15 no.3 Asunción. 2017

26. Lukes SM. Dental Decay in Southern Illinois Migrant and Seasonal Farmworkers: An Analysis of Clinical Data. The Journal of Rural Health. 2005; 21 (3): 254-25

27. Lukes SM, Miller FY. Oral health issues among migrant farmworkers. J Dent Hyg. 2002;134-140

28. Hugoson A. Caries prevalence and distribution Bjorn Thorstensson and Helene Thorstensson. The Institute for Postgraduate Dentalin 20-80-year-olds in Jonkoping , Education, Jonkoping, Sweden, in 1973, 1983, and 1993. Community Dent Oral Epidemiol 2000; 28: 90-6.

29. US Department of Health and Human Services. Healthy People 2010 (Conference Edition, in Two Volumes). Washington, DC: January 2000.

30. US Department of Health and Human Services. Oral Health in America: A Report of the Surgeon General. Rockville: US Department of Health and Human Services, National Institute of Dental and Craniofacial Research, National Institutes of Health; 2000.

31. Medina-Solís CE. Políticas de salud bucal en México: Disminuir las principales enfermedades. Una descripción. Revista Biomédica. 2006; 17:269-286.

32. Noble JM. Serum IgG antibody levels to periodontal microbiota are associated with incident Alzheimer disease. PLoS One. 2014 Dec 18; 9(12):e114959. 